\title{
Resection of the intrapancreatic bile duct preserving the pancreas
}

\author{
Armin Kolb · Jörg Kleeff • Boris Frohlich • \\ Jens Werner · Helmut Friess $\cdot$ Markus W. Büchler
}

Received: 27 February 2008/Accepted: 21 April 2008/Published online: 17 December 2008

(c) Springer 2008

\begin{abstract}
Benign neoplasms of the distal bile duct are rare, but pose a therapeutic challenge. Usually, these lesions are resected by means of ampullectomy if located in close proximity to the ampulla of Vateri or by partial pancreaticoduodenectomy if located intrapancreatic and distant from the ampulla. Here, we present a case of an intrapancreatic benign neuroendocrine tumor that was resected by performing a pancreas-preserving distal bile duct resection. First, a duodenotomy was carried out and a probe was inserted into the pancreatic duct to avoid inadvertent injury. Subsequently, the bile duct was divided proximal the lesion and dissected towards the ampulla. Pancreatic parenchyma was dissected dorsally and closed using absorbable interrupted sutures. The duodenal incision was closed, and reconstruction was performed by an endto-side hepaticojejunostomy and a Roux-Y jejunojejunostomy. The postoperative course of the patient was uneventful. In conclusion, pancreas-preserving distal bile duct resection might be an option for intrapancreatic benign lesions of the distal bile duct that would otherwise require a partial pancreaticoduodenectomy.
\end{abstract}

Keywords Bile duct resection .

Pancreaticoduodenectomy $\cdot$ Hepatectomy ·

Organ-preserving resection

A. Kolb $(\square)$ - J. Kleeff · B. Frohlich · J. Werner · H. Friess · M. W. Büchler

Department of General Surgery, University of Heidelberg, Im Neuenheimer Feld 110, 69120 Heidelberg, Germany

e-mail: armin.kolb@med.uni-heidelberg.de

\section{Introduction}

More than $95 \%$ of the tumors of the biliary system are true neoplasms. The most frequent malignancies are adenocarcinomas, of whom $60-70 \%$ arise at the bifurcation of the hepatic ducts, $20-30 \%$ in the distal common bile duct and 5-10\% intrahepatic [1]. In case of resectability malignant intrahepatic and hilar lesions frequently require standard or extended hemihepatectomies, or central liver resections. Distal bile duct cancers require partial pancreaticoduodenectomies. Benign lesions like ampullary adenomas, biliary cystadenomas, or biliary neuroendocrine tumors are quite rare [2,3]. Those tumors require complete removal as well since they have a well documented potential to become overt malignant [4]. Depending on their localization, surgical options vary: benign intrahepatic and hilar lesions are treated by formal hemihepatectomies or atypical liver resections. Hilar lesions might also be locally excised with subsequent biliodigestive anastomosis. Benign lesions in the distal common bile duct can be removed by local excision (ampullectomy), if located in close proximity to the ampulla of Vateri. Intrapancreatic lesions of the bile duct that are not accessible for ampullectomy have traditionally been resected performing a partial pancreaticoduodenectomy. Here, we describe a case of a benign neuroendocrine tumor of the distal bile duct that was removed using a pancreas-preserving distal bile duct resection.

\section{Case report}

A 64-year-old man was referred to our department with a history of increased alkaline phosphatase and gammaglutamyl-transferase levels that were detected during 


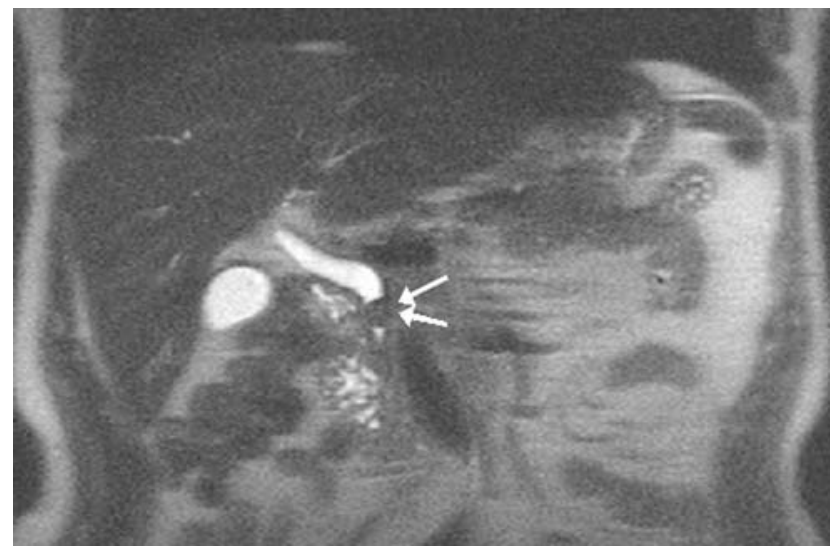

Fig. 1 MRCP demonstrating a dilated bile duct and a well-circumscribed lesion of approximately $1.5 \mathrm{~cm}$ in the distal bile duct (arrows)

routine blood examinations. Bilirubin levels and transaminases were within the normal range. The patient had no clinical symptoms, and physical examination was without any pathological findings. Ultrasound imaging demonstrated a dilated common bile duct. MRI scan revealed a $1.5 \mathrm{~cm}$ intrapancreatic lesion of the distal common bile duct without any signs of malignancy. MRCP imaging showed a biliary obstruction with a dilated common bile duct (Fig. 1). At laparotomy we found a $1.5 \mathrm{~cm}$ palpable mass apparently emanating from the distal common bile duct, and a pancreas-preserving distal bile duct resection was carried out (Figs. 2, 3). The duration of the operation was $220 \mathrm{~min}$, the intraoperative blood loss was $400 \mathrm{ml}$. The postoperative course was uneventful, and the patient was discharged on the 6th postoperative day. The histopathological report confirmed the diagnosis of a $1.4 \mathrm{~cm}$ well-differentiated benign endocrine tumor with diffuse staining for synaptophysin, neuron specific enolase (NSE), chromogranin and serotonin of the intrapancreatic bile duct (Fig. 4).

\section{Surgical technique}

The patient received singly shot antibiotic prophylaxis ( $4 \mathrm{~g}$ mezlocillin, $500 \mathrm{mg}$ metronidazole) and $200 \mu \mathrm{g}$ octreotide (Sandostatin; Novartis Pharma GmbH, Nuremberg, Germany) subcutaneously one hour before the operation. A midline incision was performed and the abdominal cavity was explored for peritoneal or hepatic lesions. Subsequently, a Kocher maneuver was carried out to mobilize the duodenum and the lesser sac was opened. Bi-manual palpation revealed a lesion in the pancreatic head region. Next, the hepatoduodenal ligament was dissected and the common bile duct was identified and looped. Subsequently, the duodenum was opened and the Papilla Vateri was identified. A probe was inserted into the main pancreatic duct to avoid any inadvertent injury. Probing of the bile duct revealed an

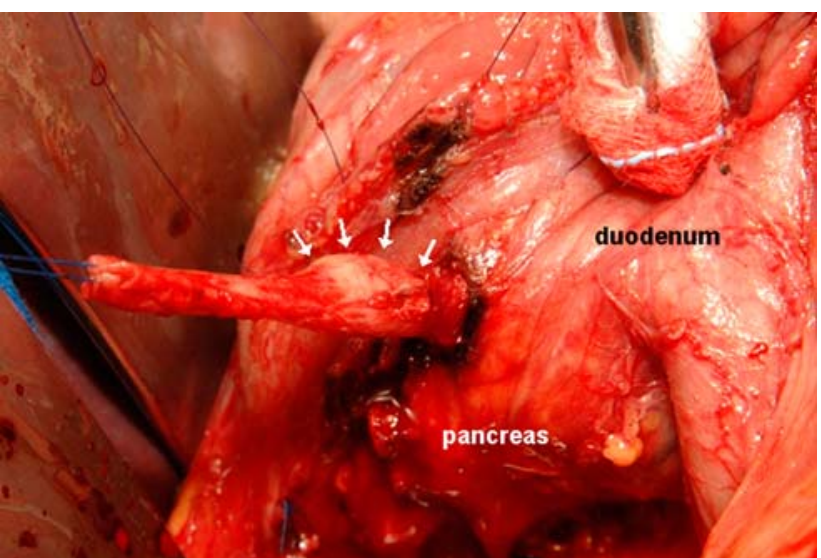

Fig. 2 Intra-operative situs demonstrating the proximally cut common bile duct with the identified lesion (arrows)

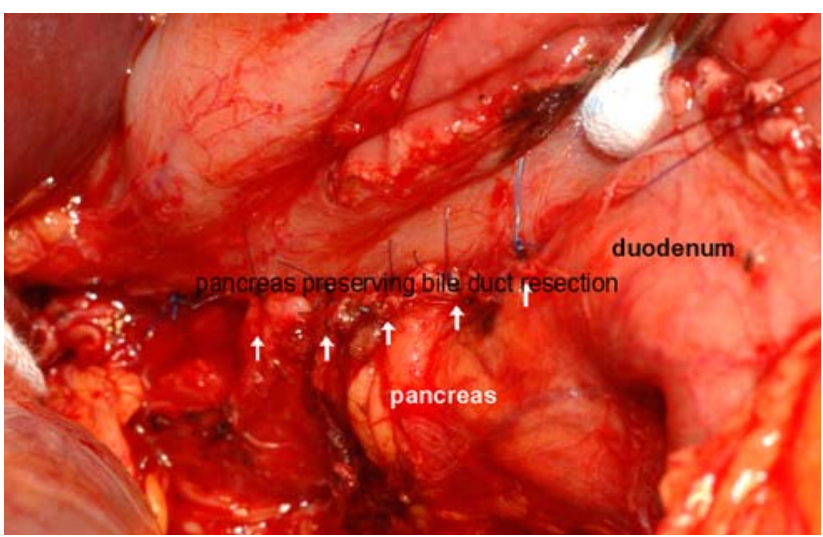

Fig. 3 Intra-operative situs demonstrating the closed dorsal pancreatic parenchyma (arrows)

intraductal lesion which was in approximately $2 \mathrm{~cm}$ distance from the papilla. Therefore, an ampullectomy was technically not feasible to resect the lesion, and the decision was made to perform a pancreas-preserving distal bile duct resection. First, a conventional cholecystectomy was performed and the common bile duct was cut $2 \mathrm{~cm}$ distal of the bifurcation. Subsequently, the bile duct was dissected towards the ampulla (Fig. 2) taking care not to injure the main pancreatic duct that was marked by the inserted probe. Pancreatic parenchyma was transected dorsally. The bile duct was dissected up-to approximately $2 \mathrm{~cm}$ distally of the identified lesion (Fig. 2, arrows), and dissected in the region of the papilla. The bile duct was closed using a 5-0 nonabsorbable monofilament suture (polybutester, Novafil, Tyco Healthcare, Mansfield, MA, USA). Special care was taken not to constrict the pancreatic duct. The dorsal pancreatic parenchyma was closed using interrupted PDS 4-0 (polydioxanone, PDS, Ethicon Inc., Piscataway, NJ, USA) sutures (Fig. 3, arrows). Subsequently, the duodenotomy was closed in a double layer fashion using again interrupted PDS 5-0 absorbable monofilament sutures. For 
Fig. 4 a Macroscopic view of the intramural benign neuroendocrine tumor located in the distal bile duct. b A welldifferentiated benign endocrine tumor with diffuse staining for synaptophysin, neuron specific enolase (NSE), chromogranin. Ki67 proliferation rate $<2 \%$
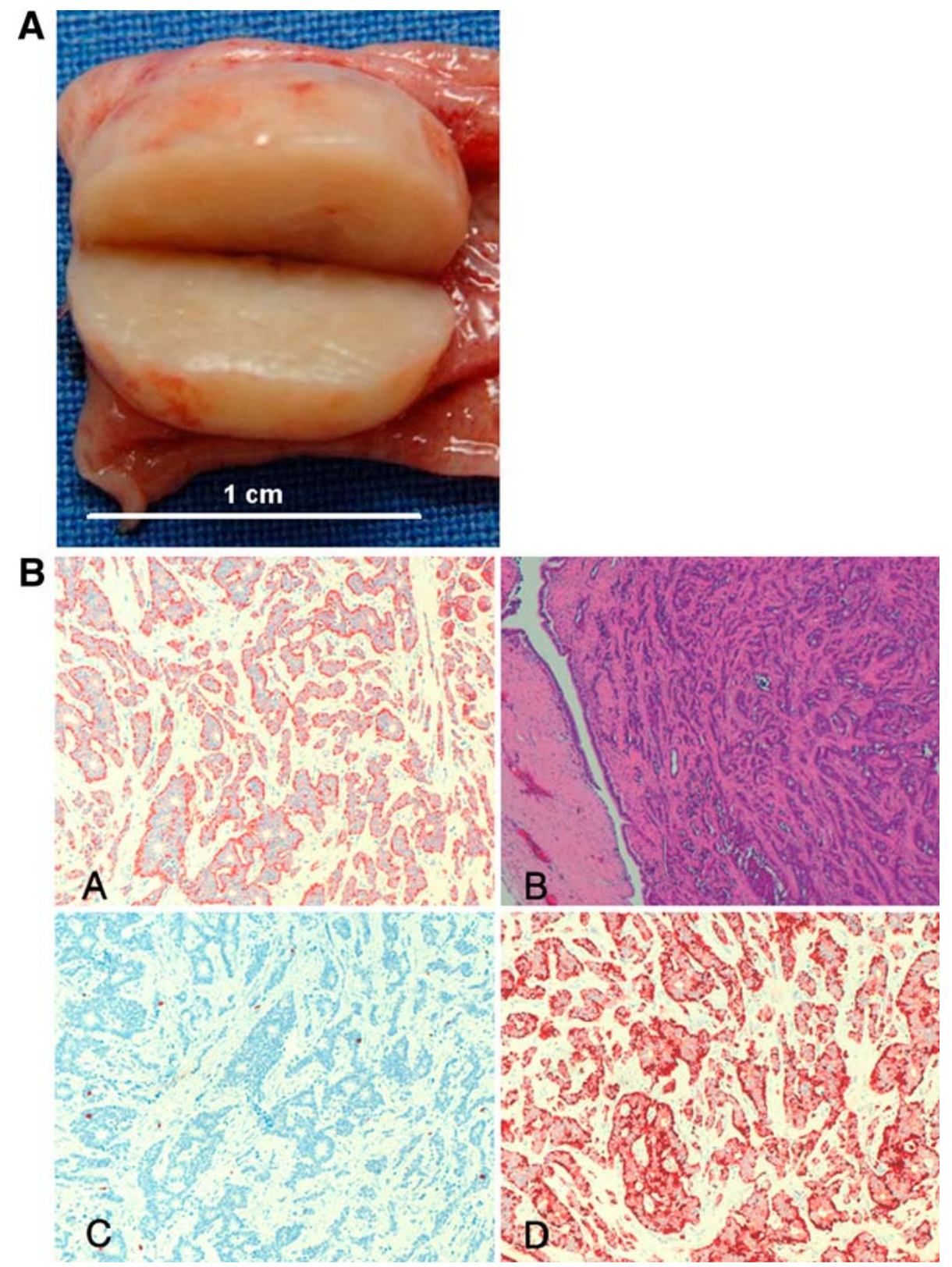

reconstruction, an end-to-side hepaticojejunostomy was carried out in single layer fashion with PDS 5-0 interrupted sutures, as well as a Roux-Y end-to-side jejunojejunostomy in a running double layer fashion with PDS 5-0. Frozen sections of the resected lesion were consistent with a benign neuroendocrine tumor. The patient received $3 \times 200 \mu \mathrm{g}$ octreotide subcutaneously for 5 days.

\section{Discussion}

Pancreatic surgery has significantly evolved in the last decades. Resectional procedures can nowadays be performed with considerable safety in experienced hands.
Nonetheless, the consequences of the loss of functional pancreatic parenchyma as well as parts of the stomach and small bowel have led to the development of an increasing number of organ-preserving approaches. These include the pylorus-preserving pancreaticoduodenectomy [5-7], the duodenum-preserving pancreatic head resection [8, 9], middle segment pancreatectomy [10-12], pancreas-preserving duodenectomy [13, 14], ampullectomy [15], and others.

In case of distal bile duct tumors, the surgical approach depends largely on the location and the underlying pathology. Malignant neoplasms of the distal bile duct are usually resected by a partial pancreaticoduodenectomy. In case of benign or borderline lesions of the ampulla or of the bile duct 
in close proximity to the ampulla, an ampullectomy can be performed as an organ-preserving procedure [16]. If the lesion is located intrapancreatic distant from the ampulla as the reported case or congenital choledochal cysts (Todani 2) $[17,18]$, surgical options are limited with partial pancreaticoduodenectomy being the surgical treatment of choice. However, for benign lesions this approach is accompanied by an unnecessary loss of functional pancreatic parenchyma as well as the duodenum as an important intestinal regulator. This approach is also associated with a considerable risk for postoperative complications. Here we describe a novel method of pancreas-preserving distal bile duct resection. A similar approach has recently been published by Kondo and co-workers [19]. However, in their approach, the pancreas was transected at the neck and the main pancreatic duct was ligated and divided just above the papilla of Vater to resect the intrapancreatic biliary system completely with the papilla of Vater. Subsequently, bilateral pancreatico-jejunostomies were created, raising the risk for postoperative complications like pancreatic fistula as was the case in the presented patient [19]. Our approach is opening the duodenum and inserting a probe to identify the bile duct, then to transect the pancreas dorsally, to resect the intrapancreatic bile duct and subsequently close the parenchyma with interrupted absorbable sutures. Although this approach carries the risk for pancreatic fistula, obviously these fistulas would be easier to control than in the case of a pancreatic anastomosis. Possibly the extent of the operation can be even decreased and done without the duodenotomy if preoperative a bile duct stent or naso-pancreatic drainage tube was placed helping to identify the bile duct. Those measures contain additional risks as inducing pancreatitis and have the disadvantage that they cannot be manipulated (inserting in the other duct) intraoperatively. Surgeons with experience in this operation might do the resection even without any aids and without opening the duodenum.

The spectrum of indications for this novel technique is narrow: benign histology has to be confirmed by frozen section, as malignant neoplasm, even if locally completely resected would require some kind of regional lymphadenectomy.

In conclusion, we describe an organ-preserving resection for benign tumors located in the distal bile duct that might be an alternative to more extensive resectional procedures in selected cases.

\section{References}

1. Nesi G, Lombardi A, Batignani G, Ficari F, Rubio CA, Tonelli F. Well-differentiated endocrine tumor of the distal common bile duct: a case study and literature review. Virchows Arch. 2006;1:104-11.

2. Davies W, Chow M, Nagorney D. Extrahepatic biliary cystadenomas and cystadenocarcinoma. Report of seven cases and review of the literature. Ann Surg. 1995;5:619-25.

3. Yeo CJ, Cameron JL, Sohn TA, Lillemoe KD, Pitt HA, Talamini MA, et al. Six hundred fifty consecutive pancreaticoduodenectomies in the 1990s: pathology, complications, and outcomes. Ann Surg. 1997;3:248-57 (discussion 257-60).

4. Wittekind C, Tannapfel A. Adenoma of the papilla and ampullapremalignant lesions? Langenbecks Arch Surg. 2001;3:172-5.

5. Diener MK, Knaebel HP, Heukaufer C, Antes G, Buchler MW, Seiler CM. A systematic review and meta-analysis of pyloruspreserving versus classical pancreaticoduodenectomy for surgical treatment of periampullary and pancreatic carcinoma. Ann Surg. 2007;2:187-200.

6. Traverso LW, Longmire WP Jr. Preservation of the pylorus in pancreaticoduodenectomy. Surg Gynecol Obstet. 1978;6:959-62.

7. Watson K. Carcinoma of the ampulla of Vater. Successful radical resection. Br J Surg. 1944;31:368-73.

8. Beger HG, Krautzberger W, Bittner R, Buchler M, Limmer J. Duodenum-preserving resection of the head of the pancreas in patients with severe chronic pancreatitis. Surgery. 1985;4:46773.

9. Muller MW, Dahmen RP, Koninger J, Di Sebastiano P, Martin D, Buchler MW, et al. The techniques of duodenum preserving head resection of the pancreas in the treatment of chronic pancreatitis. Chir Ital. 2006;3:273-83.

10. Letton AH, Wilson JP. Traumatic severance of pancreas treated by Roux-Y anastomosis. Surg Gynecol Obstet. 1959;109:473-8.

11. Muller MW, Friess H, Kleeff J, Hinz U, Wente MN, Paramythiotis D, et al. Middle segmental pancreatic resection: an option to treat benign pancreatic body lesions. Ann Surg. 2006;6:909-18 (discussion 918-20).

12. Warshaw AL, Rattner DW, Fernandez-del Castillo C, Z'Graggen K. Middle segment pancreatectomy: a novel technique for conserving pancreatic tissue. Arch Surg. 1998;3:327-31.

13. Chung RS, Church JM, vanStolk R. Pancreas-sparing duodenectomy: indications, surgical technique, and results. Surgery. 1995;3:254-9.

14. Tsiotos GG, Sarr MG. Pancreas-preserving total duodenectomy. Dig Surg. 1998;5:398-403.

15. Beger HG, Staib L, Schoenberg MH. Ampullectomy for adenoma of the papilla and ampulla of Vater. Langenbecks Arch Surg. 1998;2:190-3.

16. Paramythiotis D, Kleeff J, Wirtz M, Friess H, Buchler MW. Still any role for transduodenal local excision in tumors of the papilla of Vater? J Hepatobiliary Pancreat Surg. 2004;4:239-44.

17. Todani T, Narusue M, Watanabe Y, Tabuchi K, Okajima K. Management of congenital choledochal cyst with intrahepatic involvement. Ann Surg. 1978;3:272-80.

18. Todani T, Tabuchi K, Watanabe Y, Kobayashi T. Carcinoma arising in the wall of congenital bile duct cysts. Cancer. 1979;3:1134-41.

19. Kondo S, Hirano S, Ambo Y, Tanaka E, Morikawa T, Okushiba $\mathrm{S}$, et al. Pancreas-preserving biliary amputation with pancreatic diversion: a new surgical technique for complete resection of the intrapancreatic biliary system. Hepatogastroenterology. 2004;59: 1255-8. 